\section{Fluid dynamics of venous flow in multiple sclerosis}

\section{Trevor Tucker \\ T. Tucker Inc., Ottawa, Canada}

\section{Background}

Venous insufficiency in multiple sclerosis (MS) has often been associated with immobile valves in the tributary veins of the brachiocephalic veins (BVs) and the superior vena cava (SVC). Immobile valves have, in turn, been associated with reflux pulse flow in the tributary internal jugular (IJV), azygous (AV) and vertebral veins (VVs), causing breakdown of the blood-brain barrier (BBB) and extravasation of lymphocytes into the cerebral parenchyma. ${ }^{1,2}$

\section{Methods}

The physics of fluid dynamics is used to analyze the transition from laminar to localized chaotic flow in the BVs and SVC as represented by compliant, curved tubes with flow confluences. Chaotic flow particularly includes vortices, stagnation points and periodic reversing flow. Fluid dynamics is also applied to analyzing reflux flow patterns and standing pressure waves in the IJVs, AV, VVs and deep cerebral veins (DCVs).

\section{Results}

For typical venous structures of the BVs, SVC, IJVs, AV and VVs, fluid dynamics predicts localized vortex generation with periodic reversing flow and points of flow stagnation at curvatures, at tributary flow confluences with the BVs and SVC and at valve leaflets proximal to the confluences. Chaotic flow is associated with the development of venosclerosis plaques at such points. This analysis predicts plaque formation and valve immobility most prominently in the left IJV, secondarily the right IJV and tertiarily in the AV and VVs as observed in clinical trials. Vortex generation is also associated with valve leaflet elongation and the growth of flaps and septa as observed clinically. ${ }^{3}$ Standing wave patterns in the IJV, AV and VVs, which are dependent on venous compliances, are predicted to cause stagnant and periodic reversing flow in the DCVs, resulting in disruption of the bloodbrain barrier, also as observed clinically. ${ }^{4}$

\section{Conclusions}

Applying fluid dynamics to flow transition from laminar to chaotic in compliant tubes with curves and confluences provides substantial insight into the causality of vein scleroses and subsequent disruption of the $\mathrm{BBB}$ in $\mathrm{MS}$.
Correspondence: Trevor Tucker, Ottawa, Canada

E-mail: trevor_tucker@yahoo.com

Key words: Neurovascular diseases; meeting.

Conference presentation: $9^{\text {th }}$ Annual Meeting of the International Society of Neurovascular Disease (ISNVD), May 30 $30^{\text {th }}-31^{\text {st }}, 2019$, Ferrara, Italy.

This work is licensed under a Creative Commons Attribution 4.0 License (by-nc 4.0).

${ }^{\circ}$ Copyright: the Author(s), 2019

Licensee PAGEPress, Italy

Veins and Lymphatics 2019; 8:8430

doi:10.4081/vl.2019.8430

\section{References}

1. Menegatti E, Tessari M, Vannini ME, et al. High Resolution M-mode evaluation of jugular vein valves in patients with neurological and neurosensory disorders. Curr Neurovasc Res 2017;14:31622.

2. Zamboni P. How to objectively assess jugular primary venous obstruction. Veins and Lymphatics 2014;3:4195.

3. Tucker T. Fluid dynamics of thoracic cavity venous flow in multiple sclerosis. Med Hypotheses 2019;131:109236 (Epub ahead of print).

4. Tucker T. Fluid dynamics of cerebrospinal venous flow in multiple sclerosis. Med Hypotheses 2019;131: 109255 (Epub ahead of print). 